\title{
OBSERVATION OF VHE GAMMA RADIATION FROM HESS J1834-087/W41 WITH THE MAGIC TELESCOPE
}

J. Albert, ${ }^{1}$ E. Aliu, ${ }^{2}$ H. Anderhub,${ }^{3}$ P. Antoranz,${ }^{4}$ A. Armada,${ }^{2}$ M. Asensio, ${ }^{4}$ C. Baixeras, ${ }^{5}$ J. A. Barrio, ${ }^{4}$ M. Bartelt,${ }^{6}$ H. Bartko, ${ }^{7}$ D. Bastieri, ${ }^{8}$ S. R. Bavikadi, ${ }^{9}$ W. Bednarek, ${ }^{10}$ K. Berger, ${ }^{1}$ C. Bigongiari, ${ }^{8}$ A. Biland, ${ }^{3}$ E. Bisesi, ${ }^{9}$ R. K. Bock, ${ }^{7}$ P. Bordas, ${ }^{11}$ V. Bosch-Ramon, ${ }^{11}$ T. Bretz, ${ }^{1}$ I. Britvitch, ${ }^{3}$ M. Camara, ${ }^{4}$ E. Carmona, ${ }^{7}$ A. Chilingarian, ${ }^{12}$ S. Ciprini, ${ }^{13}$ J. A. Coarasa, ${ }^{7}$ S. Commichau, ${ }^{3}$ J. L. Contreras,${ }^{4}$ J. Cortina, ${ }^{2}$ V. Curtef, ${ }^{6}$ T. M. Dame, ${ }^{14}$ V. Danielyan, ${ }^{12}$ F. Dazzi,${ }^{8}$ A. De Angelis, ${ }^{9}$ R. De los Reyes, ${ }^{4}$ B. De Lotto, ${ }^{9}$ E. Domingo-Santamarí, ${ }^{2}$ D. Dorner, ${ }^{1}$ M. Doro, ${ }^{8}$ M. Errando, ${ }^{2}$ M. Fagiolini, ${ }^{15}$ D. Ferenc, ${ }^{16}$ E. Fernández, ${ }^{2}$ R. Firpo, ${ }^{2}$ J. Flix, ${ }^{2}$ M. V. Fonseca, ${ }^{4}$ L. Font, ${ }^{5}$ M. Fuchs, ${ }^{7}$ N. Galante, ${ }^{15}$ M. GarczarczyK, ${ }^{7}$ M. Gaug,${ }^{8}$ M. Giller,${ }^{10}$ F. Goebel, ${ }^{7}$ D. Hakobyan, ${ }^{12}$ M. Hayashida, ${ }^{7}$ T. Hengstebeck, ${ }^{17}$ D. Höhne, ${ }^{1}$ J. Hose, ${ }^{7}$ C. C. Hsu, ${ }^{7}$ P. G. Isar, ${ }^{7}$ P. Jacon, ${ }^{10}$ O. KaleKin, ${ }^{17}$ R. Kasyra, ${ }^{7}$ D. Kranich, ${ }^{3,16}$ M. LaAtiaoui, ${ }^{7}$ A. Laille, ${ }^{16}$ T. Lenisa, ${ }^{9}$ P. Liebing, ${ }^{7}$ E. Lindfors, ${ }^{13}$ S. Lombardi, ${ }^{8}$ F. Longo, ${ }^{18}$ J. López, ${ }^{2}$ M. López, ${ }^{4}$ E. Lorenz,${ }^{3,7}$ F. Lucarelli,${ }^{4}$ P. Majumdar, ${ }^{7}$ G. Maneva, ${ }^{19}$ K. Mannheim, ${ }^{1}$ O. Mansutti, ${ }^{9}$ M. Mariotti,${ }^{8}$ M. Martínez,${ }^{2}$ K. Mase, ${ }^{7}$ D. Mazin, ${ }^{7}$ C. Merck, ${ }^{7}$ M. Meucci,${ }^{15}$ M. Meyer, ${ }^{1}$ J. M. Miranda, ${ }^{4}$ R. Mirzoyan, ${ }^{7}$ S. Mizobuchi, ${ }^{7}$ A. Moralejo, ${ }^{2}$ K. Nilsson, ${ }^{13}$ E. Oña-Wilhelmi, ${ }^{2}$ R. Orduña, ${ }^{5}$ N. Otte, ${ }^{7}$ I. Oya, ${ }^{4}$ D. Paneque, ${ }^{7}$ R. Paoletti, ${ }^{15}$ J. M. Paredes, ${ }^{11}$ M. Pasanen, ${ }^{13}$ D. Pascoli,${ }^{8}$ F. Pauss,${ }^{3}$ N. Pavel,${ }^{17,20}$ R. Pegna, ${ }^{15}$ M. Persic,${ }^{21}$ L. Peruzzo, ${ }^{8}$ A. Piccioli, ${ }^{15}$ M. Poller, ${ }^{1}$ E. Prandini, ${ }^{8}$ A. Raymers, ${ }^{12}$ J. Rico, ${ }^{2}$ W. Rhode, ${ }^{6}$ M. Ribó, ${ }^{11}$ B. Riegel, ${ }^{1}$ M. Rissi, ${ }^{3}$ A. Robert, ${ }^{5}$ S. Rügamer, ${ }^{1}$ A. Saggion, ${ }^{8}$ A. Sánchez, ${ }^{5}$ P. Sartori, ${ }^{8}$ V. Scalzotto, ${ }^{8}$ V. Scapin, ${ }^{8}$ R. Schmitt, ${ }^{1}$ T. Schweizer, ${ }^{17}$ M. Shayduk, ${ }^{17}$ K. Shinozaki, ${ }^{7}$ S. N. Shore, ${ }^{22}$ N. Sidro, ${ }^{2}$ A. Sillanpä $\ddot{1},{ }^{13}$ D. Sobczynska, ${ }^{10}$ A. Stamerra, ${ }^{15}$ L. S. Stark, ${ }^{3}$ L. Takalo, ${ }^{13}$ P. Temnikov, ${ }^{19}$ D. Tescaro, ${ }^{2}$ M. Teshima, ${ }^{7}$ N. Tonello,${ }^{7}$ A. Torres, ${ }^{5}$ D. F. Torres,${ }^{2,23}$ N. Turini, ${ }^{15}$ H. Vankov, ${ }^{19}$ V. Vitale, ${ }^{9}$ R. M. Wagner, ${ }^{7}$ T. Wibig, ${ }^{10}$ W. Wittek, ${ }^{7}$ R. Zanin, ${ }^{8}$ And J. Zapatero ${ }^{5}$ Received 2006 March 17; accepted 2006 April 6; published 2006 April 27

\section{ABSTRACT}

Recently, the HESS array has reported the detection of $\gamma$-ray emission above a few hundred GeV from eight new sources located close to the Galactic plane. The source HESS J1834-087 is spatially coincident with the supernova remnant G23.3-0.3 (W41). Here we present MAGIC observations of this source, resulting in the detection of a differential $\gamma$-ray flux consistent with a power law, described as $d N_{\gamma} /(d A d t d E)=(3.7 \pm 0.6) \times$ $10^{-12}(E / \mathrm{TeV})^{-2.5 \pm 0.2} \mathrm{~cm}^{-2} \mathrm{~s}^{-1} \mathrm{TeV}^{-1}$. We confirm the extended character of this flux. We briefly discuss the observational technique used and the procedure implemented for the data analysis, and we put this detection in the perspective of the molecular environment found in the region of $\mathrm{W} 41$. We present ${ }^{13} \mathrm{CO}$ and ${ }^{12} \mathrm{CO}$ emission maps showing the existence of a massive molecular cloud in spatial superposition with the MAGIC detection.

Subject headings: gamma rays: observations — supernova remnants

\section{INTRODUCTION}

In the Galactic plane scan performed by the HESS (High Energy Stereoscopic System) Cerenkov array in 2004, with a flux sen-

\footnotetext{
${ }^{1}$ Universität Würzburg, D-97074 Würzburg, Germany.

${ }^{2}$ Institut de Física d'Altes Energies, Edifici Cn., E-08193 Bellaterra (Barcelona), Spain.

${ }^{3}$ ETH Zurich, CH-8093 Switzerland.

${ }^{4}$ Universidad Complutense, E-28040 Madrid, Spain.

${ }^{5}$ Universitat Autònoma de Barcelona, E-08193 Bellaterra, Spain.

${ }^{6}$ Universität Dortmund, D-44227 Dortmund, Germany.

${ }^{7}$ Max-Planck-Institut für Physik, D-80805 München, Germany; correspondence should be addressed to H. Bartko, hbartko@mppmu.mpg.de.

${ }^{8}$ Università di Padova and INFN, I-35131 Padua, Italy.

${ }^{9}$ Università di Udine and INFN Trieste, I-33100 Udine, Italy.

${ }^{10}$ University of Łódź, PL-90236 Lodz, Poland.

${ }^{11}$ Universitat de Barcelona, E-08028 Barcelona, Spain.

${ }^{12}$ Yerevan Physics Institute, AM-375036 Yerevan, Armenia.

${ }^{13}$ Havard-Smithsonian Center for Astrophysics, Cambridge, MA 02138.

${ }^{14}$ Università di Siena and INFN Pisa, I-53100 Siena, Italy.

${ }^{15}$ University of California, Davis, CA 95616-8677.

${ }^{16}$ Humboldt-Universität zu Berlin, D-12489 Berlin, Germany.

${ }^{17}$ Tuorla Observatory, FI-21500 Piikkiö, Finland.

${ }^{18}$ Università di Trieste and INFN Trieste, I-34100 Trieste, Italy.

${ }^{19}$ Institute for Nuclear Research and Nuclear Energy, BG-1784 Sofia, Bulgaria.

${ }^{20}$ Deceased.

${ }^{21}$ Osservatorio Astronomico and INFN Trieste, I-34100 Trieste, Italy.

${ }^{22}$ Università di Pisa and INFN Pisa, I-56126 Pisa, Italy.

${ }^{23}$ Institut de Ciències de l'Espai, E-08193 Bellaterra (Barcelona), Spain.
}

sitivity of $3 \%$ crab units for $\gamma$-rays above $200 \mathrm{GeV}$, eight sources were discovered (Aharonian et al. 2005, 2006). One of the newly detected $\gamma$-ray sources is HESS J1834-087, which is found to be, in projection, spatially coincident with the supernova remnant (SNR) G23.3-0.3 (W41). The possibility of a random correlation between the very high energy (VHE) source and SNR G23.3-0.3 was estimated to be $6 \%$ for the central region of the Galaxy (Aharonian et al. 2005). The high-energy source could also be connected to the old pulsar PSR J1833-0827 (Gaensler \& Johnston 1995), which would be energetic enough to power HESS J1834-087. However, its location 24' away from the center of HESS J1834-087 renders an association unlikely (Aharonian et al. 2005, 2006). In addition, there is also no extended pulsar wind nebula detected so far, whereas HESS J1834-087 has been found to be extended: A brightness distribution $\rho \sim \exp \left(-r^{2} / 2 \sigma^{2}\right)$ with a size $\sigma=0.09 \pm 0.02$ has been reported by HESS (Aharonian et al. 2006).

Here we present observations of HESS J1834-087 with the Major Atmospheric Gamma Imaging Cerenkov (MAGIC) telescope. We briefly discuss the observational technique used and the procedure implemented for the data analysis, derive a very high energy $\gamma$-ray spectrum of the source, and analyze it in comparison with other observations, including the molecular environment found in the region of W41. 


\section{OBSERVATIONS}

MAGIC (see, e.g., Baixeras et al. 2004 and Cortina et al. 2005 for a detailed description) is the largest single-dish imaging air Cerenkov telescope in operation. It is located on the Canary Island La Palma (N28.8, W17.8, $2200 \mathrm{~m}$ above sea level).

At La Palma, HESS J1834-087 culminates at about $37^{\circ}$ zenith angle (ZA). This ZA increases the energy threshold for MAGIC observations, but it also provides a larger effective collection area. The sky region around the location of HESS $\mathrm{J} 1834-087$ has a relatively high and nonuniform level of background light. Within a distance of $1^{\circ}$ from HESS J1834-087, there are three stars brighter than $8 \mathrm{mag}$, with the star field being brighter in the region northwest of the source. MAGIC observations were carried out in the false-source tracking (wobble) mode (Fomin et al. 1994). The sky directions (W1, W2) to be tracked are such that in the camera, the sky brightness distribution relative to $\mathrm{W} 1$ is similar to the one relative to $\mathrm{W} 2$. In both cases, the source direction is 0.4 offset from the camera center. These two tracking positions are shown as white stars in Figure 1. For each tracking position, two background control regions are used, which are located symmetrically to the source region (denoted by the central white circle) with respect to the camera center. During wobble mode data taking, $50 \%$ of the data is taken at $\mathrm{W} 1$, and $50 \%$ at $\mathrm{W} 2$, switching (wobbling) between the two directions every 30 minutes. This observation mode allowed for a reliable background estimation least affected by the medium-scale ZA and the inhomogeneous star field. HESS J1834-087 was observed for a total of $20 \mathrm{hr}$ in the period 2005 August-September $\left(\mathrm{ZA} \leq 45^{\circ}\right)$. In total, about 12 million triggers have been recorded.

\section{DATA ANALYSIS}

The data analysis was carried out using the standard MAGIC analysis and reconstruction software (Bretz \& Wagner 2003), the first step of which involves the calibration of the raw data (Gaug et al. 2005). It follows the general steps presented in Albert et al. (2006a, 2006b): After calibration, image cleaning tail cuts of 10 photoelectrons for image core pixels and 5 photoelectrons for boundary pixels have been applied (see, e.g., Fegan 1997). These tail cuts are accordingly scaled for the larger size of the outer pixels of the MAGIC camera. The camera images are parameterized by image parameters (Hillas 1985). In this analysis, the Random Forest method (see Bock et al. 2004 and Breiman 2001 for a detailed description) was applied for the $\gamma /$ hadron separation (for a review see, e.g., Fegan 1997) and the energy estimation. For the training of the Random Forest, a sample of Monte Carlo-generated $\gamma$-showers (Majumdar et al. 2005) was used together with about $1 \%$ of randomly selected events from the measured wobble data. The Monte Carlo (MC) $\gamma$-showers were generated between $35^{\circ}$ and $45^{\circ} \mathrm{ZA}$ with energies between $10 \mathrm{GeV}$ and $30 \mathrm{TeV}$ with a SIZE distribution equal to one of the selected data events for the training. The source-position-independent image parameters SIZE, WIDTH, LENGTH, CONC (Hillas 1985), and the third moment of the photoelectron distribution along the major image axis were selected to parameterize the shower images. After the training, the Random Forest method allows us to calculate for every event a parameter, the so-called hadronness, which is a measure of the probability that the event is not $\gamma$ like. The $\gamma$-sample is defined by selecting showers with a hadronness below a specified value. An independent sample of MC $\gamma$-showers was used to determine the cut efficiency.

The analysis at similar ZAs was developed and verified using Crab Nebula data taken in 2005 September (see also Albert et

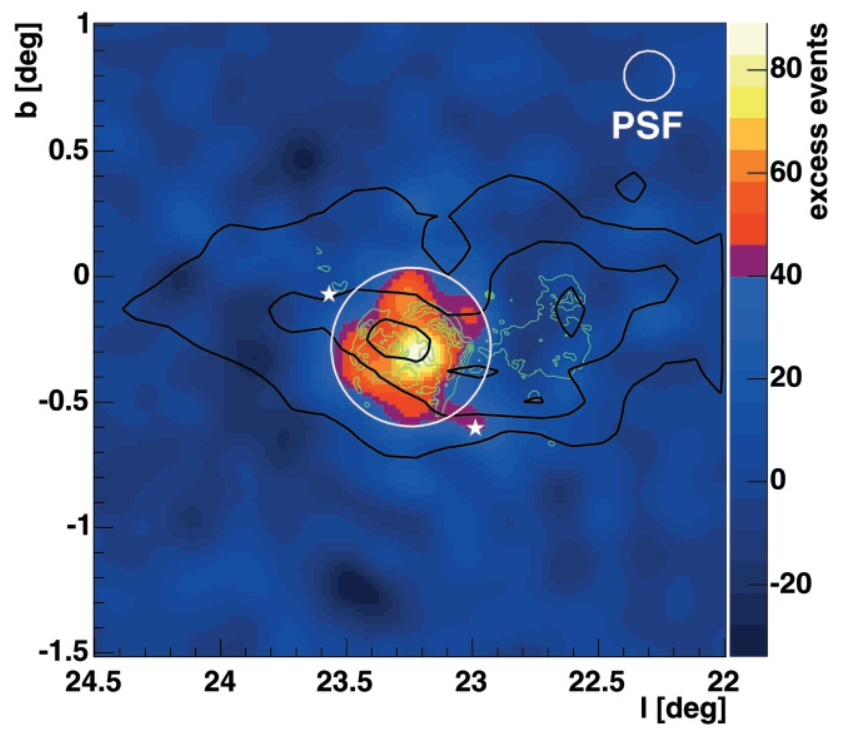

FIG. 1.-Sky maps of $\gamma$-ray candidate events (background-subtracted) in the directions of HESS J1834-087 for an energy threshold of about $250 \mathrm{GeV}$. The source is clearly extended with respect to the MAGIC PSF. The two white stars denote the tracking positions in the wobble mode. Overlaid are ${ }^{12} \mathrm{CO}$ emission contours (black) from Dame et al. (2001) and contours of $90 \mathrm{~cm}$ VLA radio data from White et al. (2005; green). The ${ }^{12} \mathrm{CO}$ contours are at 25/50/75 $\mathrm{K} \mathrm{km} \mathrm{s}^{-1}$, integrated from 70 to $85 \mathrm{~km} \mathrm{~s}^{-1}$ in velocity, the range that best defines the molecular cloud associated with W41. The contours of the radio emission are at 0.04/0.19/0.34/0.49/0.64/0.79 $\mathrm{Jy}$ beam $^{-1}$, chosen for showing both SNRs G22.7-0.2 and G23.3-0.3 at the same time. Clearly, there is no superposition with SNR G22.7-0.2. The central white circle denotes the source region integrated for the spectral analysis.

al. 2006c). The Crab energy spectrum, as determined by our studies, was consistent with measurements from other experiments (see dashed line in Fig. 4 below).

For each event, the arrival direction of the primary $\gamma$-ray candidate in sky coordinates is estimated using the DISP method (Fomin et al. 1994; Lessard et al. 2001; DomingoSantamaria et al. 2005). To select a subset of events with superior angular resolution, a conservative lower SIZE cut of 200 photoelectrons is applied. The corresponding analysis energy threshold is about $250 \mathrm{GeV}$.

Figure 1 shows the sky map of $\gamma$-ray candidates (backgroundsubtracted; see, e.g., Rowell 2003) from the direction of HESS J1834-087. It is folded with a two-dimensional Gaussian with a standard deviation of 0.072 and a maximum of one. The MAGIC $\gamma$-ray point-spread function (PSF; standard deviation of a twodimensional Gaussian fit to the nonfolded brightness profile of a point source) is $0.1 \pm 0.01$. The folding of the sky map serves to increase the signal-to-noise ratio by smoothing out statistical fluctuations. However, it somewhat degrades the spatial resolution. The sky map is overlaid with contours of $90 \mathrm{~cm}$ VLA radio data (green) from White et al. $(2005 ; 20 \mathrm{~cm}$ radio data from the same reference are overlaid in the following figures) and ${ }^{12} \mathrm{CO}$ emission contours from Dame et al. (2001; black), integrated in the velocity range $70-85 \mathrm{~km} \mathrm{~s}^{-1}$, the range that best defines the molecular cloud associated with W41. The MAGIC excess is centered at (R.A., decl.) $=\left(18^{\mathrm{h}} 34^{\mathrm{m}} 27^{\mathrm{s}},-8^{\circ} 42^{\prime} 40^{\prime \prime}\right)$. The statistical error is 0.5 , and the systematic pointing uncertainty is estimated to be $2^{\prime}$ (see Bretz et al. 2003). A fit of a two-dimensional Gaussian brightness profile to the nonfolded sky map yields, after subtraction of the MAGIC $\gamma$-ray PSF in quadrature, an intrinsic source extension of $\sigma=0.14 \pm 0.04$ (the extension reported by HESS is $0.09 \pm 0.02$; Aharonian et al. 2006). Both position and extension coincide well with the shell-type SNR G23.3-0.3 (W41). 


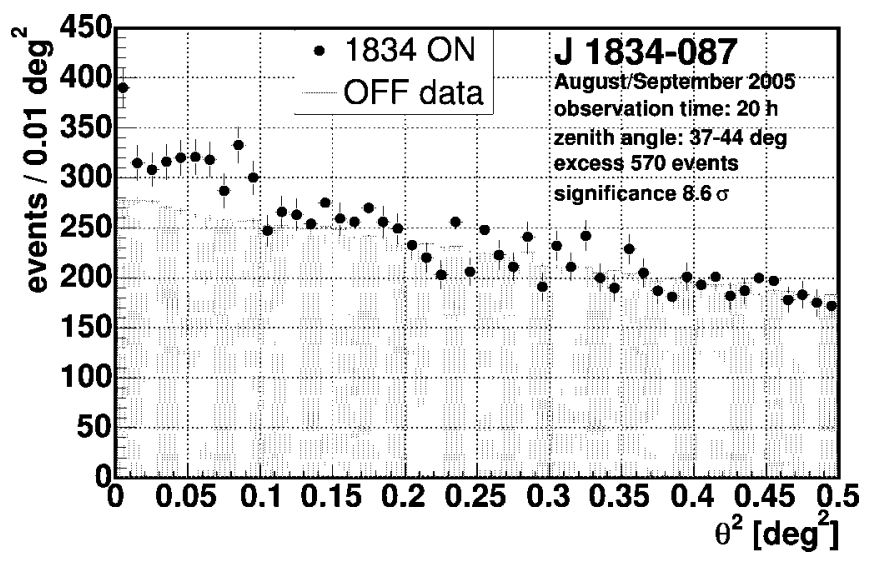

FIG. 2.-Distributions of $\theta^{2}$-values for the source (filled circles) and background control region (shaded histogram) for an energy threshold of about $250 \mathrm{GeV}$. Here it is also seen that the source is clearly extended with respect to the MAGIC PSF $\left(\sigma^{2} \sim 0.01 \mathrm{deg}^{2}\right)$.

Figure 2 shows the distribution of the squared angular distance, $\theta^{2}$, between the reconstructed shower direction and the excess center. The observed excess in the direction of HESS J1834-087 has a significance of $8.6 \sigma$ for $\theta^{2} \leq 0.1 \mathrm{deg}^{2}$. Figure 3 shows images of HESS J1834-087 with three different lower cuts on SIZE (200, 300, and 600 photoelectrons), corresponding to energy thresholds of about 250, 360, and $590 \mathrm{GeV}$, respectively. As in Figure 1, the background-subtracted sky maps are folded with a two-dimensional Gaussian, but here the color scale shows directly the excess significance. The total observed excess significance for $\theta^{2} \leq 0.1 \mathrm{deg}^{2}$ (corresponding to the sky region inside the central white circle of Fig. 1) are $8.6 \sigma, 7.8 \sigma$, and $7.3 \sigma$ for the three lower cuts on SIZE. Overlaid are contours of $20 \mathrm{~cm}$ VLA radio data (green) from White et al. (2005) and ${ }^{13} \mathrm{CO}$ emission contours (black) from Jackson et al. (2006). The contours of the radio emission are at $0.0035 \mathrm{Jy} \mathrm{beam}^{-1}$. The ${ }^{13} \mathrm{CO}$ contours are integrated from 70 to $85 \mathrm{~km} \mathrm{~s}^{-1}$ in velocity, as was the ${ }^{12} \mathrm{CO}$ data in Figure 1. For all three SIZE cuts, the MAGIC PSF is about $0{ }^{\circ} .1$, and the source position, extension, and morphology stay roughly constant. The characteristics of the MAGIC observation are compatible within errors with the measurement of HESS (Aharonian et al. 2006).

For the spectral analysis, a sky region with a maximum angular distance of $\theta^{2}=0.1 \mathrm{deg}^{2}$ around the excess center (indicated by the white circle in Fig. 1) has been integrated. Figure 4 shows the reconstructed very high energy $\gamma$-ray

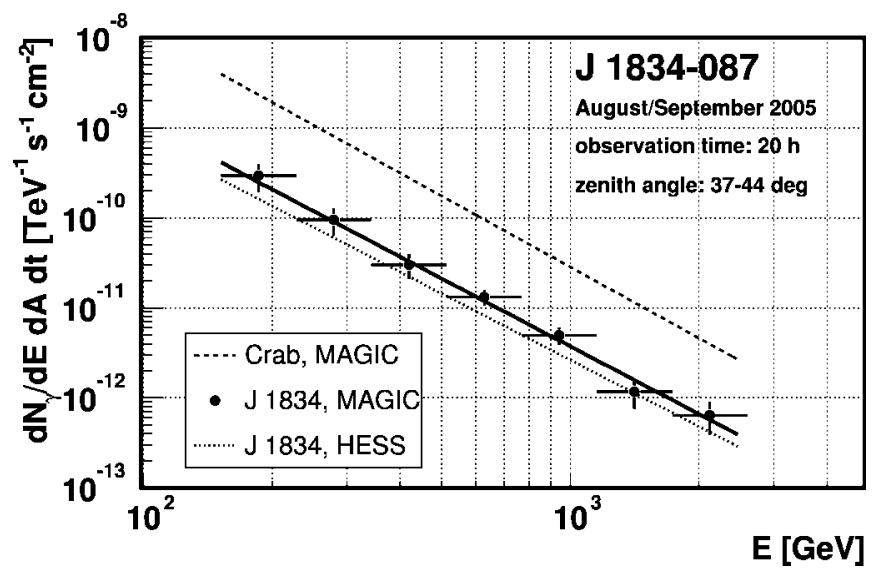

FIG. 4.-VHE $\gamma$-ray spectrum of HESS J1834-087 (statistical errors only). The solid line shows the result of a power-law fit to the data points. The dotted line shows the result of the HESS collaboration (Aharonian et al. 2006). The dashed line shows the spectrum of the Crab Nebula as measured by MAGIC (Wagner et al. 2005).

spectrum $\left[d N_{\gamma} /\left(d E_{\gamma} d A d t\right)\right.$ vs. true $\left.E_{\gamma}\right]$ of HESS J1834-087 after correcting (unfolding) for the instrumental energy resolution (Anykeev et al. 1991). The horizontal bars indicate the bin size in energy; the marker is placed in the bin center on a logarithmic scale. The solid line shows the result of a forward unfolding procedure: A simple power-law spectrum is fitted to the measured spectrum $\left[d N_{\gamma} /\left(d E_{\gamma} d A d t\right)\right.$ vs. estimated $E_{\gamma}$ ], taking the full instrumental energy migration (true $E_{\gamma}$ vs. estimated $E_{\gamma}$ ) into account as described in Mizobuchi et al. (2005). The result is given by $\left(\chi^{2} / \mathrm{dof}=\right.$ $7.4 / 7) d N_{\gamma} /(d A d t d E)=(3.7 \pm 0.6) \times 10^{-12}(E / T e V)^{-2.5 \pm 0.2}$ $\mathrm{cm}^{-2} \mathrm{~s}^{-1} \mathrm{TeV}^{-1}$. The quoted errors are statistical. The systematic error is estimated to be $35 \%$ in the flux level determination and 0.2 in the spectral index (see also Albert et al. 2006b). Within the observation time (weeks), no flux variations exceeding the measurement errors have been observed. Also, the flux is compatible within errors with the measurement of HESS made 1 year earlier.

\section{DISCUSSION AND CONCLUDING REMARKS}

SNRs as gamma-ray sources have been extensively discussed in the past (e.g., see Torres et al. 2003 for a review). Due to the spatial coincidence between the VHE $\gamma$-ray source and the SNR G23.3-0.3 (W41), this SNR appears to be the natural
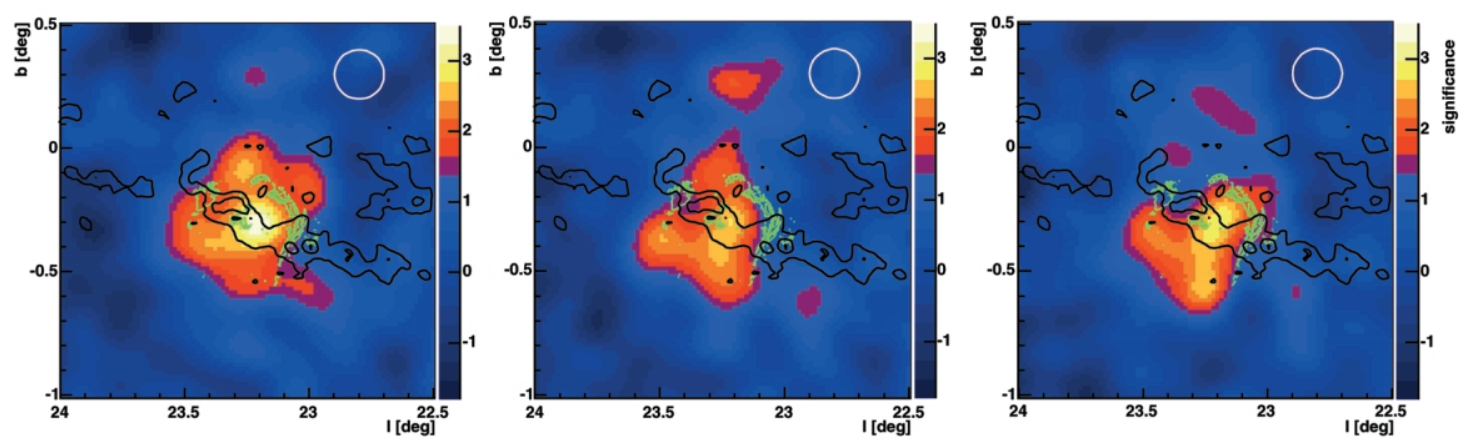

FIG. 3.-Morphology of HESS J1834-087 above three different lower cuts in SIZE (200, 300, and 600 photoelectrons), corresponding to energy thresholds of 250,360, and $590 \mathrm{GeV}$, respectively. The color scale shows the excess significance. Overlaid are contours of $20 \mathrm{~cm}$ VLA radio data (green) from White et al. (2005) and ${ }^{13} \mathrm{CO}$ emission contours (black) from Jackson et al. (2006). The contours of the radio emission are at $0.0035 \mathrm{Jy}$ beam ${ }^{-1}$. The ${ }^{13} \mathrm{CO}$ contours are at $10 /$ $20 / 30 \mathrm{~K} \mathrm{~km} \mathrm{~s}^{-1}$, integrated from 70 to $85 \mathrm{~km} \mathrm{~s}^{-1}$ in velocity, as was the ${ }^{12} \mathrm{CO}$ data in Fig. 1 . The white circle indicates the MAGIC PSF, which is about 0.1 for all three lower SIZE cuts. 
candidate for generating the observed $\gamma$-ray emission. W41 is an asymmetric shell-type SNR, with a diameter of $27^{\prime}$. It is included in Green's catalog (Green 2004), and has a spectral index of 0.5 and a flux density of $70 \mathrm{Jy}$ at $1 \mathrm{GHz}$. It was mapped in radio with the VLA array at $330 \mathrm{MHz}$ (Kassim 1992), and at 20 and $90 \mathrm{~cm}$ (see White et al. 2005), following earlier studies (see, e.g., Ariskin \& Berulis 1970, Shaver \& Goss 1970, and references therein). It is partially overlapping with SNR G22.7-0.2 (see, e.g., Fig. 9 of Kassim 1992), although the latter is not in coincidence with the peak of the very high energy source (see Fig. 1). No Chandra and XMM observations of W41 are publicly available yet.

W41 was associated with a very large molecular complex called "[23,78]" in Dame et al. (1986). There, it was concluded that there are probably two large clouds blended at that position in $(l, b, v)$ space, one on the near side of the $4 \mathrm{kpc}$ arm and another on the far side of the Scutum Arm. The giant molecular cloud associated with W41 is best defined by integrating the $\mathrm{CO}$ emission from 70 to $85 \mathrm{~km} \mathrm{~s}^{-1}$ in velocity. The $\mathrm{CO}$ emission peaks near $l=$ $23.3, b=-0.3, v=78 \mathrm{~km} \mathrm{~s}^{-1}$; the near kinematic distance of this peak is $4.9 \mathrm{kpc}$. The peak is marked by the central black contour in Figure 1, which lies very close to the VHE source. The total $\mathrm{H}_{2}$ mass of the cloud, computed over the ranges $l=22^{\circ}-$ $24.25, b=-0.75-0.5$, and $v=70-85 \mathrm{~km} \mathrm{~s}^{-1}$, and assuming a distance of $4.9 \mathrm{kpc}$, is $2.1 \times 10^{6} M_{\odot}$. This mass is necessarily an upper limit since, as mentioned above, there is certainly an emission contribution from unrelated gas at the far kinematic distance. Still, the $\mathrm{CO}$ peak is so strong and well defined that it most likely arises from gas primarily at one location, near the VHE source, rather than being a random blend of emissions from the near and far distances. The total $\mathrm{H}_{2}$ mass of the $\mathrm{CO}$ emission peak in Figure 1 (computed over the region $l=23^{\circ} .2-23^{\circ} 4, b=-0.35$ to $-0^{\circ} 15, v=70-85 \mathrm{~km} \mathrm{~s}^{-1}$ ) is $8.8 \times 10^{4} M_{\odot}$. The higher resolution ${ }^{13} \mathrm{CO}$ map in Figure 3, which was derived from the recently completed Galactic Ring Survey (Jackson et al. 2006), confirms that the VHE source lies toward the local enhancement of molecular material, i.e., a giant molecular cloud.

At $5 \mathrm{kpc}$, the luminosity of HESS J1834-087 between $250 \mathrm{GeV}$ and a few $\mathrm{TeV}$ is about $5 \times 10^{34} \mathrm{ergs} \mathrm{s}^{-1}$, similar to the luminosity of HESS J1813-178 (Albert et al. 2006a) if that source is considered associated with SNR G12.8-0.0 at a distance of $\sim 4 \mathrm{kpc}$. We note, however, that J1813-178 has been found to be nearly pointlike, whereas, in the present case, a significant extension is observed. The $\gamma$-ray spectrum of HESS J1834-087 is steeper than that of $\mathrm{J} 1813-178$. From the observed $\gamma$-ray luminosity, and assuming an acceleration efficiency of hadrons on the order of $3 \%$ and a supernova power of $10^{51} \mathrm{ergs}$, the required density of matter in the $\gamma$-ray production region for hadrons to be mainly responsible for the observed radiation can be estimated from the formula $L_{\gamma} \sim$ (velocity of light) $\times$ (density) $\times$ (efficiency of acceleration $) \times$ (supernova power $) \times(p-p$ cross section $) \times$ ( $\gamma p-p$ inelasticity), and it is of about $\sim 11 \mathrm{~cm}^{-3}$ (see Torres et al. 2003 and references therein). With the extension of HESS J1834-087, and the gas mass found to be in the innermost contour of the CO map, i.e., in close superposition with the very high energy source, there is enough mass to generate the high-energy radiation hadronically, even if only part of the gas is interacting with the SNR shock.

All in all, the observation of HESS J1834-087 using the MAGIC telescope confirms a new very high energy extended $\gamma$-ray source in the Galactic plane. A reasonably large data set was collected from observations at medium-scale zenith angles to infer the spectrum of this source up to energies of a few TeV. Above $200 \mathrm{GeV}$, the differential energy spectrum can be fitted with a power law of slope $\Gamma=-2.5 \pm 0.2$. The results of the independent observations of the HESS and MAGIC telescopes are in agreement within errors concerning the level of flux, the spectral shape, the morphology, and the extension of the source. The coincidence of the VHE $\gamma$-ray source with SNR G23.3-0.3 (W41) suggests that this SNR is a natural counterpart to HESS J1834-087, and although the mechanism responsible for the high-energy radiation remains yet to be clarified, a massive molecular cloud has been identified in the region.

We would like to thank the IAC for the excellent working conditions at the Observatory de los Muchachos in La Palma. The support of the German BMBF and MPG, the Italian INFN, and the Spanish CICYT is gratefully acknowledged. This work was also supported by ETH research grant TH 34/04 3 and the Polish MNiI grant 1P03D01028. This Letter is dedicated to the memory of Nicolaj Pavel.

\section{REFERENCES}

Aharonian, F., et al. 2005, Science, 307, 1938 2006, ApJ, 636, 777

Albert, J., et al. 2006a, ApJ, 637, L41 2006b, ApJ, 638, L101 2006c, ApJ, 639, 761

Anykeev, V. B., Spiridonov, A. A., \& Zhigunov, V. B. 1991, Nucl. Instrum. Methods Phys. Res. A, 303, 350

Ariskin, V. I., \& Berulis, I. I. 1970, Soviet Astron., 13, 883

Baixeras, C., et al., 2004, Nucl. Instrum. Methods Phys. Res. A, 518, 188

Bock, R. K., et al. 2004, Nucl. Instrum. Methods Phys. Res. A, 516, 511

Breiman, L. 2001, Machine Learning, 45, 5

Bretz, T., \& Wagner, R. 2003, Proc. 28th Int. Cosmic Ray Conf. (Tsukuba), 2947

Bretz, T., et al. 2003, Proc. 28th Int. Cosmic Ray Conf. (Tsukuba), 2943

Cortina, J., et al. 2005, Proc. 29th Int. Cosmic Ray Conf. (Pune), 5, 359

Dame, T. M., Elmegreen, B. G., Cohen, R. S., \& Thaddeus, P. 1986, ApJ, 305,892

Dame, T. M., Hartmann, D., \& Thaddeus, P. 2001, ApJ, 547, 792

Domingo-Santamaria, E., et al. 2005, Proc. 29th Int. Cosmic Ray Conf. (Pune), 5,363

Fegan, D. J. 1997, J. Phys. G, 23, 1013
Fomin, V. P., Stepanian, A. A., Lamb, R. C., Lewis, D. A., Punch, M., \& Weekes, T. C. 1994, Astropart. Phys., 2, 137

Gaensler, B. M., \& Johnston, S. 1995, MNRAS, 275, L73

Gaug, M., Bartko, H., Cortina, J., \& Rico, J. 2005, Proc. 29th Int. Cosmic Ray Conf. (Pune), 5, 375

Green, D. A. 2004, Bull. Astron. Soc. India, 32, 335

Hillas, A. M. 1985, Proc. 19th Int. Cosmic Ray Conf. (La Jolla), 3, 445

Jackson, J. M., et al. 2006, ApJS, 163, 145

Kassim, N. E. 1992, AJ, 103, 943

Lessard, R. W., Buckley, J. H., Connaughton, V., \& Le Bohec, S. 2001, Astropart. Phys., 15, 1

Majumdar, P., Moralejo, A., Bigongiari, C., Blanch, O., \& Sobczyńska, D. 2005, Proc. 29th Int. Cosmic Ray Conf. (Pune), 5, 203

Mizobuchi, S., et al. 2005, Proc. 29th Int. Cosmic Ray Conf. (Pune), 5, 323 Rowell, G. P. 2003, A\&A, 410, 389

Shaver P. A., \& Goss, W. M. 1970, Australian J. Phys. Astrophys. Suppl., 14, 133

Torres, D. F., Romero, G. E., Dame, T. M., Combi, J. A., \& Butt, Y. M. 2003, Phys. Rep., 382, 303

Wagner, R., et al. 2005, Proc. 29th Int. Cosmic Ray Conf. (Pune), 4, 163

White, R. L., Becker, R. H., \& Helfand, D. J. 2005, AJ, 130, 586 